\title{
El retraso en el desarrollo: una respuesta educativa*
}

\section{Climent Giné**}

\author{
Departament d'Ensenyament de la Generalitat de Catalunya
}

Nos proponemos con el presente trabajo ahondar en algunos aspectos tanto de orden conceptual como metodológico que a nuestro entender pueden contribuir al diseño básico del proceso educativo de las personas con retraso en el desarrollo desde y a través de la institución escolar. Es evidente que un objetivo de estas características encierra una gran complejidad puesto que intervienen muchas y distintas variables, tanto por la diversidad de enfoques con que tradicionalmente se ha abordado el tema como por la estrecha relación que guarda con opciones que deberían analizarse desde la sociología y desde la política.

En efecto, la educación especial, expresión con que genéricamente se refiere la atención educativa que la sociedad presta a los niños y niñas con retraso en el desarrollo a través de la escuela, ha sido históricamente objeto de enfoques que en ocasiones han puesto el énfasis casi exclusivamente en aspectos médicos, rehabilitadores, o bien psicológicos e incluso también enfoques puramente asistenciales sin prácticamente otros objetivos que el cuidado físico y la aocupación» durante el tiempo en que los niños permanecían en la escuela. Por otra parte, y es importante señalarlo, la educación especial ha sido y es tributaria de los planteamientos que la sociedad ha ido progresivamente asumiendo en relación a la salud mental, en general, y a lo que podríamos llamar marginación social; toda la política educativa actual en relación a la integración, por poner un ejemplo, es un claro exponente de lo que acabamos de señalar.

Como parece lógico, puesto que de lo contrario nos desviaríamos de nuestro objetivo, no vamos a entrar en el análisis de la bondad o no de tales opciones ni de las razones que históricamente las explican y en algunos casos justifican, sin que ello deba interpretarse como postura de neutralidad; a lo largo de la exposición se irá decantando, esperamos que con claridad, nuestra propia posición con sus opciones básicas.

Nos proponemos, pues, analizar y describir aquellos elementos que a nuestro entender, tanto a nivel conceptual como por las consecuencias que tienen en la prestación del servicio, mejor contribución al diseño de un modelo educativo en relación al retraso en el desarrollo; entiéndase que con aretraso en el desarrollos nos referiremos genéricamente al conjunto de trastornos que alteran el normal desarrollo de un sujeto ocasionando, en mayor o menor grado, algún tipo de déficit o limitación. Evidentemente, todo modelo educativo es tributario de un conjunto de presupuestos y de opciones entre los cuales ocupan un

* El presente trabajo corresponde a la ponencia que el autor desarrolló en el ciclo: Psicología strastornos de Conducta en la infancias en el marco de los V Cursos de Verano en San Sebastián, organizados por la Universidad del País Vasco. 25-27 de Agosto de 1986.

** Dirección de autor: Paseo de la Reina Elisenda de Montcada, 13. 08034 Barcelona. 
lugar destacado la visión que se tiene del educando y las expectativas en relación a qué tipo de necesidades, y en qué grado, deben ser satisfechas en y a través de la escuela; es por esta razón que nos ocuparemos en primer lugar de exponer lo que entendemos por retraso en el desarrollo y el concepto de educación especial que se deriva.

\section{Concepto de retraso en el desarrollo}

De acuerdo con Bijou (1975) entendemos como más adecuado utilizar el término retraso en el desarrollo para referirnos con carácter general a determinados problemas en el proceso de desarrollo de algunos niños, como señalábamos antes. Varios han sido los términos utilizados por las disciplinas (medicina, psicología, pedagogía) que tradicionalmente se han ocupado de este tipo de alteraciones para referirse tanto al problema en sí como a los sujetos afectados; no se trata de introducir un modismo más, cosa que por otro lado carecería de sentido dado el amplio uso que de este término se hace en otras áreas lingüísticas $e$ incluso entre nosotros. Si nos parece importante señalarlo es porque, a nuestro entender, pone de relieve con mayor facilidad que otros términos su naturaleza funcional y su carácter descriptivo, nunca explicativo. En efecto, como señalábamos ampliamente en un trabajo anterior (Giné, Espuñes y Carasa, 1985) uno de los aspectos que han tenido una fuerte incidencia en la concepción y explicación del retraso en el desarrollo, de graves consecuencias tanto a nivel familiar, como en relación a las expectativas sociales ante el problema, como incluso también en el ámbito profesional, ha sido el hecho de que la utilización de términos como deficiencia mental, subnormalidad, e incluso dislexia o ergastenia..., inicialmente con fines tan sólo descriptivos, progresivamente ha ido dando paso a la consolidación de una variable hipotética («deficiencia mentals,...) la cual por sí misma causaría y explicaría el comportamiento de determinados individuos y, en general, el fenómeno psicológico de lo que llamamos retraso en el desarrollo. El peso social de esta creencia, y sus consecuencias, son suficientemente evidentes para no ser necesario insistir en ello.

Al hablar de retraso en el desarrollo hacemos hincapié en su naturaleza funcional y en la múltiple determinación del problema, evitando esquematizar su interpretación en términos simples de causa-efecto, como así sucede con demasiada frecuencia. Nos parece importante señalar que el término retraso en el desarrollo debe considerarse únicamente como un concepto descriptivo, no explicativo, que encierra un alto grado de relatividad; en efecto, el desarrollo puede ser estimulado, acelerado o compensado mediante el diseño de condiciones favorables ajustadas a las condiciones particulares de un niño determinado, tanto a nivel personal como del ambiente que le rodea (familia, escuela...).

Vemos pues que la reflexión sobre el retraso nos remite necesariamente al concepto mismo de desarrollo. Como señalan Bijou (1975) y Galindo (1984), «el desarrollo psicológico debe ser entendido como la adquisición sucesiva por parte del individuo de nuevas formas de conducta cada vez más complejas cualitativa y cuantitativamente, en función de factores orgánicos, físicos y sociales». Así pues, desde una perspectiva psicológica, el estudio del desarrollo nos lleva a centrarnos en las formas de conducta del individuo que presentan niveles de organización y complejidad crecientes y diferentcs entre sí a lo largo del proceso de desarrollo.

Siguiendo a Ribes $(1980,1985)$ entendemos la conducta como la interacción históricamente construida entre el organismo (el individuo en desarrollo) y su ambiente físico y social; esta interacción entre el individuo y su ambiente es, por naturaleza, dinámica y fundamentalmente está determinada por el equipo 
biológico de base; por las características del ambiente: tanto el más próximo (la familia y la escuela, la calidad de las relaciones interpersonales, y el entorno físico) como el entorno socio-económico y cultural de la sociedad en que vive; y por la historia de las interacciones a lo largo de la vida del individuo.

Así pues, la conducta de un individuo determinado va sufriendo a lo largo del tiempo una serie de cambios, adquiriendo nuevas formas, cada vez más complejas, que son fruto del concurso de los factores que acabamos de señalar; y en esto consiste el desarrollo psicológico. Desde esta perspectiva, podemos afirmar que la presencia o no de determinados factores, no sólo cuantitativa sino cualitativamente, se convierte en condiciones que necesariamente van a incidir en el desarrollo, sea favoreciéndolo sea limitándolo.

Aunque no vamos a entrar en el análisis de cada uno de estos tres grupos de variables que inciden en el desarrollo, sí que deseamos enfatizar un aspecto. De hecho, es suficientemente conocido que cuando existe un déficit en el equipo biológico de base de un niño, su relación con el ambiente físico y social se ve radicalmente modificada; en general podríamos decir que en la medida en que se produce un enlentecimiento en el desarrollo se produce una pérdida de posibilidades de aprovechar las oportunidades que lo facilitarían. Pero hay más; lo que con frecuencia sucede es que el ambiente próximo que rodea al niño, que cuenta con algún tipo de déficit, en los primeros meses y años de su vida, se ve global y profundamente alterado. El contacto físico, la relación afectiva de los padres y familiares, la interacción positiva, el juego, las oportunidades para ejercitar tal o cual habilidad, ...se tornan faltos de ilusión, escépticos y cada vez más escasos. Es decir, aunque estemos plenamente de acuerdo con Roca (1983) cuando afirma que usean cuales sean las características orgánicas de un individuo al nacer, estas se constituyen en factores disposicionales», nunca como causas fatalistas de un determinado proceso de desarrollo, lo que en realidad se da con frecuencia, tal como acabamos de apuntar, es una historia de intracción tremendamente deprivada, alterada y, en consecuencia, empobrecedora.

Si nos detuviéramos en analizar, y estuviéramos realmente en condiciones de poderlo hacer con un cierto rigor, secuencias de a vida de niños (sanos y con problemas) nos daríamos cuenta de hasta qué punto el nivel de desarrollo alcanzado es consecuencia en gran medida de la presencia o ausencia de condiciones favorables y oportunidades, sobre todo en relación a la interacción personal.

Así pues, cuando existe un déficit en el equipo biológico de base de un individuo y/o se ve sometido a condiciones sociales y físicas desfavorables, el resultado es un enlentecimiento en la adquisición de nuevas formas de conducta; este enlentecimiento lo denominamos retraso en el desarrollo.

Es desde esta perspectiva, es decir, desde las condiciones presentes en la historia de las interacciones, a lo largo de la vida de un niño, cuya cualidad va a favorecer $o$ a dificultar el desarrollo, que a nuestro entender conviene analizar el problema del retraso, incidir en su prevención y diseñar este apartado, que siempre el desarrollo, y por lo tanto el retraso, se produce como consecuencia de la interacción de las variables señaladas y difícilmente podemos identificar una, de forma aislada, como causa directa.

\section{La educación especial: concepto de necesidades educativas especiales}

Hasta aquí hemos procurado explicitar los presupuestos teóricos básicos con que contemplamos el proceso de desarrollo de todo ser humano, las variables que en él inciden y que, en consecuencia, condicionan este complejo fenómeno 
al que hemos llamado retraso en el desarrollo, que afecta a determinados individuos. Aunque hasta ahora nos hayamos referido a las edades más tempranas, es obvio que cuanto hemos señalado se extiende a todo el proceso; es decir a las distintas etapas de la vida del ser humano. Evidentemente cada etapa tiene unas peculiaridades, unas necesidades que cubrir en orden a favorecer el armónico y pleno crecimiento de todos los individuos; estaremos de acuerdo si afirmamos que precisamente los fines de la educación son promover este crecimiento, tanto en el orden personal como social y que la institución a la que la sociedd encomienda tan delicada tarea es la escuela. Cuando la atención educativa, como decíamos al principio, se dirige a los niños y niñas con retraso en el desarrollo a través de la escuela, recibe el nombre de educación especial.

Quisiéramos, a continuación, exponer el concepto de educación especial que manejamos porque va a informarnos de las opciones que en este campo hemos tomado al tiempo que va a precisar los rasgos generales que han de caracterizar la respuesta de la escuela para satisfacer las necesidades de los alumnos con problemas.

Nuestra concepción de la educación especial tiene su origen, por un lado, en los presupuestos teóricos en relación al desarrollo, que hemos señalado, y, por otro lado, en la transformación que en la última década se ha ido produciendo en este campo, y en consecuencia en la educación en general, contrastada con la experiencia del trabajo en el aula. Conviene insistir en que si exponemos lo que entendemos por educación especial es tan sólo para acotar nuestra posición, no porque consideremos que sea este un tema cerrado; al contrario, el debate sigue, y ha de seguir, abierto implicando a los padres, los profesionales, la Universidad y, por supuesto, los responsables de la política educativa.

Como decíamos, desde hace algunos años muchos países han ido tomando conciencia de la situación de marginación en la que se hallaban - y hallandiversos colectivos, entre los que se encontraban las personas con retraso en el desarrollo, sobre todo por las limitaciones al pleno desarrollo que tales condiciones inevitablemente comportaban. Este proceso ha supuesto cambios conceptuales importantes en el ámbito educativo (Brennan, 1982) en el que se ha ido abriendo camino una concepción de escuela particularmente atenta a su función social y educativa de favorecer el desarrollo de todos los alumnos, sean cuales sean sus características individuales y las de su entorno. Dos hitos de este proceso, históricamente reconocidos como fundamentales, son la legislación italiana en relación a la integración escolar de los alumnos con retraso en el desarrollo y el conocido Warnock Report (1978); entre nosotros, debemos referitnos al Plan Nacional de Educación Especial (1978), la L.I.S.M.I. (1982) y los decretos que la han desarrollado en el ámbito del Estado y de las distintas Comunidades Autónomas.

Esta nueva visión de escuela abierta a la diversidad, que en nuestro caso ha hecho suya el Departament d'Ensenyament de la Generalitat de Catalunya tanto a nivel conceptual como desde la propia normativa (Decret 117/84, de 17 de abril sobre ordenación especial radicalmente diferente de la acepción tradicional del término; «se trata de un concepto amplio de educación especial que se ubica en el marco de un modelo de actuación fundamentalmente educativo, y por lo tanto, atento a todo aquello que es preciso (posible) proporcionar al alumno para favorecer su desarrollo personal, básicamente en y a partir del ámbito escolar» (Ruiz y Giné, 1986).

En síntesis ase pretende que la escuela responda a las necesidades educativas de todos los alumnos, sea cual sea su grado de especificidad y tanto si se derivan, en mayor o menor medida, de hándicaps, déficits, etc., como de cualqueir otro factor social, familiar, escolar, etc. que incide en el crecimiento personals (Ruiz i Giné, 1986). 
Este concepto amplio de educación especial que contrasta con la categorización de los alumnos en base al handicap, a que estamos tan acostumbrados, nos lleva al concepto de necesidades educativas especiales, término acuñado por el mencionado Warnock Report (1978).

Nuestra Constitución consagra el derecho a la educación de todos los ciudadanos; en consecuencia, todos los niños participan de unas mismas necesidades: la provisión de aquellos medios y recursos de orden material y personal que les permitan acceder a los fines generales de la educación en ejercicio del derecho correspondiente. El citado Warnock Report (1978) afirma: aSi hay acuerdo sobre los objetivos de la educación, entonces una «necesidad educativas se establece en términos de aquello que es esencial para el logro de estos objetivoss...; y más adelante «las necesidades de cada niño le son específicas: son aquello que necesita si se desea que, individualmente progrese. Por tanto, las necesidades educativas conforman, en líneas generales, un continuum».

Ruiz, en un reciente trabajo (1986) sintetiza los rasgos comunes de este planteamiento:

a) el énfasis en las necesidades educativas concebidas como acontinuum» que comprende desde las más generales a las más particulares y específicas.

b) el concepto de necesidades educativas «especiales» como el hecho de precisar ayudas pedagógicas específicas para el logro de los fines de la educación.

c) la asimilación de estas ayudas pedagógicas específicas a recursos personales, materiales y técnicos provinientes del marco educativo.

En síntesis, «un enfoque plenamente educativo de las necesidades individuales (diferencias) nos conducen a un modelo de actuación más atento a la identificación y determinación de las ayudas pedagógicas que es necesario proporcionar al alumno en relación a sus características que es necesario proporcionar al alumno en relación a sus características individuales, que no a considerarlo sujeto de aplicación de un sinfin de clasificaciones que desde diferentes perspectivas y disciplinas ponen el énfasis en el handicap. De esta manera, un enfoque profundamente educativo nos obliga a considerar los factores de hándicap como uno de los indicadores que nos ayudarán a precisar el tipo de necesidad educativa, pero sólo como un factor en un proceso que, por el hecho de ser educativo, incluye muchos otros elementos de interación referidos al medio familiar, escolar y social de cada alumno» (Ruiz i Giné, 1986).

Vemos, pues, un amplio acuerdo entre los fundamentos psicológico y pedagógico del modelo que proponemos; por un lado, el concepto de retraso en el desarrollo pone el énfasis en su carácter funcional: en las condiciones presentes en la historia de interacción del niño que van a estimular y favorecer su crecimiento personal y social, y, por otro lado, el concepto de educación especial con el que operamos («necesidades educativas especiales») se traduce en una descripción positiva sobre la actuación educativa que cada niño requiere, de acuerdo con sus características y circunstancias personales, para acceder a los fines generales de la educación que, como hemos señalado, no son otros que el máximo crecimiento personal y social.

Quizás alguno se preguntará cómo es que hablando de retraso en el desarrollo y educación especial, en ningún momento nos hayamos referido al concepto de inteligencia, como parecería obligado; evidentemente no se trata de un descuido y es consecuencia del posicionamiento teórico que sostenemos. El desarrollo de la inteligencia es el desarrollo de la competencia del individuo en relación con su medio, mediante el proceso educativo; es pues, una parte del proceso de desarrollo del individuo. Como señala Ribes (1984), ael desarrollo de la inteligencia consiste en la educación de la inteligencia, pero en educación no entendida como la suma de contenidos formales, sino como ejercicio de actividades funcionales con respecto a dichos contenidos. La competencia se adquie- 
re enseñando el «cómo» y no «el qués. Por ello el desarrollo de la inteligencia debe concebirse como la estructuración educativa de formas genéricas de interacción funcional con el ambiente».

\section{Elementos que configuran la respuesta educativa}

Muchas son las variables que intervienen en el proceso educativo y que por lo tanto deben ser contempladas en la estructuración de toda respuesta que pretenda cubrir eficazmente las necesidades de todos los alumnos durante su etapa escolar. Además, no debemos perder de vista que, con ser importante la escuela, la vida del niño discurre en otros ámbitos, que no vamos a abordar en este trabajo, de tanta o mayor trascendencia para su desarrollo como son la familia y la comunidad; es por lo tanto obligado, so pena de ignorar la globalidad del niño, contemplar la vida escolar en total y constante interacción con los otros ámbitos.

De las distintas variables, vamos a centrarnos en el estudio tan sólo de aquellas que conceptualmente, a nuestro entender, mejor pueden contribuir al diseño de un modelo educativo en relación al retraso en el desarrollo, coherente con los presupuestos de orden teórico y metodológico que hemos establecido. Nos referimos a la valoración del retraso, a la oferta curricular y a la propia existencia de proyecto educativo.

\section{Valoración del retraso en el desarrollo}

Decíamos que el concepto de retraso en el desarrollo es eminentemente funcional y en consecuencia la forma de evaluarlo ha de ser igualmente funcional. Dos han sido las constantes con que tradicionalmente se ha venido abordando el tema. En primer lugar, la prácticamente exclusiva utilización del modelo médico en la valoración - adiagnósticos - no sólo de los factores orgánicos que a menudo están presentes, junto a otros factores, en el retraso en el desarrollo, sino que su utilización se extendía a todas las áreas del desarrollo sin cuestionar su pertinencia. La utilización sistemática del modelo médico en la valoración del retraso es una cuestión compleja y que fácilmente se presta a la polémica; de todas maneras, el estudio de que ha sido objeto, en los últimos años, desde diferentes disciplinas ha puesto en evidencia sus limitaciones y la fragilidad de algunos planteamientos y conclusiones. Sin embargo, hay total acuerdo en considerar la enorme importancia que ha tenido - y todavía tiene en algunos ámbitos-, junto con su repercusión social, para los padres, para la escuela o para el propio afectado. No es momento de ahondar en el análisis de la prevalencia del modelo médico - con las excepciones que deban hacerse- ni de su trascendencia; señalemos tan sólo, un par de aspectos que han caracterizado dicha utilización; por un lado, el énfasis en la categorización, de los sujetos y de los problemas, acompañada de un gran esfuerzo para dotarse de una amplia taxonomía en base a criterios cuya relación con el problema era, a menudo, más que discutible; por otro lado, la extensión del modelo no sólo al diagnóstico sino al tratamiento de los sujetos afectados. Una consecuencia inevitable, entre otras, es que la etiqueta, con que siempre se concluia el diagnóstico, acuñada inicialmente para describir una determinada disfunción se convertía poco a poco en explicativa y en «causa del comportamiento de la persona con retraso.

En segundo lugar, era, y a menudo es todavía, prácticamente obligado para la valoración del retraso recurrir a las pruebas de inteligencia y/o similares. No 
es necesario insistir en el desprestigio de las prácticas psicométricas, a raíz de las críticas que desde muy diversos sectores se le han formulado, en relación al retraso sobre todo porque se han revelado de escasísima utilidad para plantear la acción educativa. Es éste también un tema que merecería mayor atención, pero en general su uso se inscribe también en el modelo médico, del que es tributario: con las pruebas se mide ala deficiencias, se exploran las «potencialidades internas», se detecta «el déficit» y/o sla patologías y normalmente se traduce en sesiones de reeducación y/o psicoterapia, sean del signo que fueren; pero raras veces su uso trasciende los propios condicionantes metodológicos para situarse en la arena en la que discurre la vida de estos niños, es decir en las condiciones reales de desarrollo.

Realmente la valoración del retraso es un elemento crucial para cualquier aproximación a este complejo problema y, por supuesto, para el diseño del tipo de respuesta que va a darse. Es por esta razón que las opciones que en este campo se tomen revisten una singular importancia por la trascendencia que a nivel metodológico encierran, no sólo en relación a los instrumentos que van a utilizarse $-\mathrm{y}$ que en alguna medida prefiguran los resultados - sino en el establecimiento de las actuaciones posteriores.

En coherencia con los presupuestos técnicos que hemos expuesto, dos son los rasgos que a nuestro entender deben caracterizar el proceso de valoración del retraso. En primer lugar, el proceso de valoración ha de servirnos para identificar funcionalmente las necesidades del individuo en las distintas áreas de desarrollo, poniendo en relación su nivel de competencia con las condiciones y oportunidades presentes en el proceso de desarrollo (en la historia de interacción) en la perspectiva de que las variables de tipo biológico deben ser consideradas funcionalmente como factores disposicionales; en este sentido, el fruto de la valoración no puede ser otro que el diseño de estrategias y actuaciones encaminadas a facilitar el desarrollo del individuo, disponiendo oportunidades, modificando condiciones o fortaleciendo prácticas convenientes.

En segundo lugar, la naturaleza del retraso en el desarrollo y del proceso de valoración exigen un enfoque interdisciplinar. Distintas son las variables y distintas las disciplinas científicas que tienen por objeto su estudio, pero sólo desde una visión global y contrastada puede asegurarse una correcta percepción del problema. Es este un tema difícil, entre otras razones por la falta de interdisciplinariedad en nuestra propia formación, por el estatus social de las profesiones, etc.; sin embargo, está fuera de discusión que un análisis honesto del retraso exige el concurso de diversos profesionales, de acuerdo con las variables que inciden, y este es uno de los retos más serios que hoy nos plantea el trabajo profesional. No quisiéramos dejar este punto sin poner de relieve que los ambientes reales de vida del niño, con los profesionales - y en su caso los padresque lo tienen bajo su responsabilidad, estén presentes en el proceso de valoración; en este sentido debemos afirmar que es impresdindible la participación del maestro en esta tarea, cuando del ámbito escolar se trate.

En síntesis, centrándonos en la escuela, el proceso de valoración debe permitirnos identificar, como señala Ruiz (1986), cuáles son las necesidades educativas del alumno y cuál es su grado de especificidad, precisando elementos como: a) tipo y grado de especificidad de las adecuaciones curriculares que va a ser necesario establecer en relación a un alumno determinado, como por ejemplo, selección y priorización de los contenidos y objetivos, temporalización y priorización de los contenidos y objetivos, temporalización, estrategias acordes con el perfil del alumno, etc.; y b) medios de acceso al curriculum que será necesario facilitar al alumno, como por ejemplo, los medios personales, los materiales, etc. Estas cuestiones, junto con las más generales que afectan el desarrollo en geneal y el proceso educativo en particular como son los aspectos afectivos, 
la relación interpersonal, social, etc. van a permitirnos con mayor facilidad formular las actuaciones educativas adecuadas.

Guillespie-Silver et al., (1980) señalan, de forma esquemática que la valoración en la escuela debe servirnos básicamente para determinar:

a) si las características de un determinado alumno le hacen tributario de actuaciones eductivas diferenciadas.

b) aquello que realmente tiene bien establecido y aquello que está poco o nada establecido.

c) el estilo de aprendizaje del alumno.

d) el nivel de competencia (rendimiento) en cada una de las áreas.

e) el progreso que experimenta hacia el logro de los objetivos que en cada caso se propongan.

f) la eficacia del plan seguido.

\section{La oferta curricular}

Desde una perspectiva básicamente educativa, en la cual nos hemos ido moviendo y que se desprende del marco teórico expuesto, el sistema curricular aparece como elemento central para el diseño de la respuesta a las diferentes necesidades educativas de todos los alumnos, en la medida en que integra el aconjunto de elementos que informan, en el ámbito escolar, sobre qué, cómo y cuándo enseñar a los alumnos en función de los fines generales de la educación» (Ruiz i Giné, 1986) y de acuerdo con sus características.

En el trabajo que acabamos de citar, y al cual nos iremos refiriendo entresacando algunos párrafos, realizamos un análi8sis y descripción de la respuesta a las necesidades educativas especiales desde el curriculum escolar a partir de la propuesta que en este sentido ha asumido el Departament d'Ensenyament de la Generalitat de Catalunya.

En efecto, un enfoque curricular acorde con una concepción de escuela abierta a la diversidad, a la que nos referíamos anteriormente, exige poder disponer de un marco curricular común para todos los alumnos independientemente de la modalidad o nivel educativo. El Departament d'Ensenyament, situándose en esta perspectiva y después de un largo proceso ha optado por un único marco curricular para toda la enseñanza obligatoria (Coll, 1986). En síntesis esta opción se formula en los siguientes términos:

a) un único marco curricular para toda la enseñanza obligatoria; ello implica que las orientaciones y programas de todos los ciclos de la enseñanza obligatoria se elaboren a partir de un modelo formal y conceptual único...; también significa que las orientaciones específicas para los alumnos con necesidades educativas especiales deberán formularse a partir del mismo marco curricular, marco que debe contemplar la diversidad.

b) unas orientaciones y programas abiertos y flexibles... Ello se concreta técnicamente en un modelo curricular caracterizado por: 1) una estructura que prevé diferentes niveles de concreción de las intenciones educativas a partir de los objetivos generales de cada ciclo; y 2) unos elementos para la detección y valoración de las necesidades educativas y de determinación de las actuaciones educativas en función de estas mismass.

En este contexto, las necesidades educativas especiales a que debe poder responderse desde el curriculum escolar, las podemos agrupar, siguiendo a Ruiz (1986) en dos grupos.

El primer grupo de necesidades educativas serían presentadas por aquellos alumnos para los cuales es necesario diseñar actuaciones educativas que impliquen fundamentalmente modificaciones curriculares en relación a los conteni- 
dos y a los objetivos. Estas modificaciones se refieren a: aspectos de temporalización y/o secunciación de objetivos y contenidos; priorización de determinados contenidos y objetivos complementarios o alternativos a los enunciados con carácter general en las áreas curriculares.

El segundo grupo de necesidades educativas se referirían a los medios - de menos a más específicos- de acceso al curriculum escolar; estos medios podrían concretarse, de acuerdo con la valoración del alumno, en: la disposición de situaciones educativas específicas: trabajo individual, en grupo, en casa,...; determinados recursos personales: profesor de apoyo, logopedas,...; materiales específicos: instrumentos que faciliten la comunicación vocal y escrita,...; condiciones para el acceso físico a la escuela: adaptaciones arquitectónicas, equipamiento específico...

El documento escolar en el que se describe y concreta el conjunto de elementos que integran la respuesta educativa en relación a un determinado alumno, recibe el nombre de programa de desarrollo individual, (P.D.I.); aunque no podemos detenernos en su estudio, digamos que, en líneas generales, se trata de un instrumento cuya función es orientar las actuaciones educativas encaminadas a promover el desarrollo global del alumno, a partir de la valoración que de sus necesidades educativas se haya llevado a cabo. Es, pues, fundamentalmente un «plan de acción que puede ayudarnos a evaluar el progreso, a modificarlo y a comunicarnos con los padres y otros profesionales» (Gillespie -Silver, 1980).

\section{El proyecto educativo}

No podemos olvidar que el marco en el que tiene lugar el diálogo educativo, la actuación pedagógica, es una institución: la escuela, en la que intervienen los alumnos, los maestros, los padres y, en muchos casos la comunidad en la que se halla inserta. Tal como ha puesto de manifiesto la psicología, y últimamente con mayor intensidad, toda institución tiene su propia vida, sus normas de juego, unos fines propios y en su seno se manifiestan diversos intereses de acuerdo con los distintos grupos que la conforman; el estudio de los fenómenos de tipo psicológico que se producen en la vida de las instituciones, desde el consenso al conflicto, ha logrado identificar determinadas constantes que permiten predecir y explicar en gran medida su comportamiento.

Es lógico pensar, y así sucede en realidad, que la institución como tal juega un papel de primera magnitud en el diálogo educativo que pueda establecerse, por lo que todas las cuestiones que puedan incidir en su mejor funcionamiento, es decir en que la escuela cumpla la misión educativa, que tiene encomendada, de forma óptima tiene una gran trascendencia y en ningñun caso deben subestimarse o simplemente ignorarse.

Evidentemente, existe una amplia gama de resortes e instrumentos que pueden facilitar en funcionamiento armónico de las instituciones $y$, en concreto, de la que nos ocupa, la escuela; a tal fin constituye un elemento crucial la existencia de un proyecto educativo, tanto por lo que supone su elaboración como por la trascendencia al regular aspectos importantes de la vida de la institución e, incluso, al poder dirimir situaciones de conflicto. Así lo ha entendido últimamente la Administración Educativa, regulando su elaboración y convirtiendo el tema en uno de los tópicos del momento; no es que se trate hoy en día de una novedad, ya que la tradición pedagógica de muchas escuelas había pusto de manifiesto su importancia y la propia Administración, en otros términos, lo había exigido ya con anterioridad; lo que ciertamente es nuevo y de trascendencia es el reconocimiento de quienes han de tomar parte en su elaboración 
y el papel que se les otorga en su aprobación y seguimiento. Lo cierto es que todos habremos tenido ocasión de comprobar que tras un trabajo pedagógico serio y comprometido ha existido siempre un proyecto educativo coherente.

Dejando a un lado la dinámica de las Instituciones y sin pretender analizar a fondo los distintos factores que entraña un proyecto educativo ni definirlo de forma exhaustiva, lo cual por sí solo merecería un trabajo aparte, sí que podemos afirmar que, a nuestro entender, el proyecto educativo debe establecer al menos acuerdos respecto a tres núcleos de cuestiones fundamentales: a elementos conceptuales en relación al propio concepto de escuela y de educación; a los educandos con sus derechos y deberes; a la función social...; b) elementos de organización escolar; y c) elementos de participación y relación con la comunidad escolar, sobre todo los padres.

Por lo que tespecta a los elementos conceptuales, estamos convencidos que de las decisiones que en este campo puedan tomarse son de enorme importancia a pesar de que puedan parecer demasiado teóricas o filosóficas; un proyecto educativo que asuma la visión de escuela y de las necesidades educativas de los alumnos, como la que hemos señalado en el apartado segundo al referinos al concepto de educación especial, hará posible el establecimiento del modelo educativo que propugnamos. En efecto, una escuela capaz de no rechazar a determinados alumnos por sus mayores o menores diferencias, capaz de responder diferencialmente a las necesidades individuales, entraña una particular visión de la educación y de la interacción en la escuela que evidentemente tiene repercusiones directas en el trabajo pedagógico diario, tanto por lo que respecta a la organización del trabajo como a la programación y evaluación como también a la valoración de los problemas que surjan. No vamos a insistir en ello para no repetir aspectos ya señalados, pero las consecuencias de según que opciones se van a hacer sentir no sólo en relación a los alumnos con algún tipo de problema sino en todos los alumnos; es decir, lo que se debate no es si procede o no «integrar» a un alumno con retraso en el desarrollo sino que está en juego algo mucho más profundo, como es la calidad educativa, el estilo de trabajo, en definitiva, cómo la escuela va a responder a las evidentes diferencias presentes en todo grupo humano. Si bien es cierto, que a nivel conceptual existen también otras cuestiones sobre las que pronunciarse y que aquí no vamos a abordar, es igualmente cierto que la opción que al respecto se tome, de alguna manera, va a teñir todas las demás.

Sin duda, en la organización escolar se agrupan la mayoría de decisiones que afectan al funcionamiento del centro, desde el horario a la organización del aula y a la toma de determinadas decisiones. A continuación quisiéramos llamar la atención sobre algunas cuestiones sobre las que es necesario llegar a acuerdos y que, a nuestro entender, parecen más pertinentes en relación al tipo de modelo que proponemos. Como es lógico, de lo que se trata es de que la organización sea permeable a los principios que hemos ido exponiendo a lo largo del trabajo, y en este sentido nos parece oportuno insistir en:

1. El proceso de identificación y valoración de los problemas que aparecen en el proceso enseñanza/aprendizaje de los alumnos incluso los derivados del retraso en el desarrollo; es ésta una cuestión delicada, tal como ya hemos descrito ampliamente con anterioridad, y que a menudo puede exigir el concurso de profesionales ajenos al centro. Conviene no renunciar a la importante función del maestro en este cometido porque la observación sistemática, la recogida de datos, el esfuerzo en describir sin categorizar, el énfasis en las necesidades y en lo que el niño es capaz de hacer y no en la «patología» o el «déficit» sitúa en inmejorable condición la aportación del maestro. Debe, pues, lograrse acuerdos sobre los objetivos y procedimientos a seguir por el Claustro en la identificación y valoración de los problemas de acuerdo con los principios señalados. 
2. Asimismo, el proyecto educativo debe prever la existencia de diferentes niveles de concreción de las intenciones educativas a partir de los objetivos generales de cada ciclo/área, de acuerdo con la valoración de las necesidades educativas de un alumno determinado; ello supone, como señalábamos, la posibilidad de adecuar el curriculum al momento de desarrollo y características del alumno.

3. Como complementación al punto anterior, la respuesta a las necesidades educativas supone también la provisión de determinados medios que permitan al alumno el logro de los objetivos que para él se hayan fijado; entre éstos, los que están más al alcance del maestro, son los referidos a la organización de la clase: distribución del espacio, del tiempo, el diseño de estrategias didácticas, etc.

4. El proyecto educativo debe también incluir acuerdos sobre la evaluación; de ésta una tarea muy frecuente y de enorme trascendencia en la vida de la escuela, de los alumnos y de sus familias. Deben precisarse los objetivos que se proponen y las actividades de evaluación pertinentes tanto para los alumnos a los que se les haya adaptado el curriculum como para todos los demás. En la medida de lo posible y dentro del respeto a la normativa vigente debe procurarse utilizar pruebas de evaluación referidas a criterio, es decir, referidas al progreso del alumno en relación al logro de los objetivos que tiene establecidos. Asimismo también, las actividades de evaluación deben permitir al Claustro recabar información sobre la eficacia del sistema y de la metodología utilizada tanto con carácter general como la más específica.

5. El proyecto educativo debe establecer claramente espacios de tiempo regulares para el trabajo en equipo del Claustro (ciclo, área...) y para la formación permanente; una tarea de esta trascendencia no puede dejarse ni a la buena voluntad ni al azar de las posibilidades. Debe fijarse la metodología y dinámica de las sesiones de trabajo, preveyendo la posibilidad que el grupo ciclo...) pueda enriquecerse con la participación de un especialista en relación a un alumno o a un tema determinado. Sólo de esta manera es posible consolidar un trabajo pedagógico de calidad y la continuidad del proceso educativo de maestro a maestro y de año en año.

Finalmente, el proyecto educativo debe asegurar la participación de los padres y la relación con la comunidad; es éste un tema rico en valores educativos que no puede reducirse a la anécdota de la salida por el barrio o a participar en una fiesta popular. Sin embargo, querríamos llamar la atención en relación a los padres; no sólo por la necesaria participación, por otro lado contemplada en la legislación vigente, sino porque a nuestro entender la dimensión educativa del centro debe de alguna manera contemplar por un lado las anecesidades de formación de los padres en relación a los problemas del desarrollo, tanto a nivel individual como de grupo, y por otro lado su incorporación a determinados programas sin cuyo concurso los objetivos educativos perseguidos difícilmente llegarían a lograrse. 


\section{Resumen}

Este articulo pretende analizar y describir aquellos elementos que, tanto a nivel conceptual como por las conse. cuencias que tienen en la práctica diaria, mejor contribuyen al diseño de un modelo fundamentalmente educativo en relación a las personas con retraso en el desarrollo. Se abordan, en primer lugar, los fundamentos psicológico y pedagógico del modelo propuesto: la naturaleza funcional del retraso en el desarrollo y el concepto de educación especial (anecesidades educativas especialess) que se traduce en una descripción positiva de la actuación educativa que cada niño requiere. A continuación, se describen los elementos de mayor trascendencia en el diseño de la respuesta educativa: la valoración del retraso en el desamollo, la oferta curricular y las características del proyecto educativo.

\section{Summary}

This paper intends to analize and to describe the elements that - both at a conceptual level and according to their consequences in every day practice - can make the best contribution to the design of a basically educative model for developmentally retarded persons. The paper meets first the psychological and pedagogical basis of the proposed model: the fonctional features of development retard and the special education concept (uspecial educational needss) that results in a positive account of requirements. The paper then describes the most significant elements in the educative answer design: the developmental retarded assesment, the schools curricula and the educative project traits.

\section{Referencias}

BJjOu, S. W. (1975). Teoría e investigación sobre el retardo mental (en el desarrollo). En S.W. Bijou y D.M. Baer Psicologia del desarrollo infantil, vol. 2. Méjico: Trillas.

Brennan. W. (1982). Changing Special Education. Milton Keynes: The Open University Press.

COLL. C. (1986). Mare curricular per a l'ensenyament obligatori. Barcelona: Generalitat de Catalunya.

Decret $117 / 84$ de 17 d'abril sobre ordenació de l'educació especial per a la seva integració en el sistema educatiu ordinari. DOGC de 18-5-84.

GaINDO. E. (1984). El concepto de retardo en el desarrollo y sus implicaciones para la enseñanza y el entrenamiento. En 1. Galguera, G. Hinojosa y E. Galindo, El retardo en el desamollo. Teoría y präctica. Méjico: Trillas.

GIIESPIE-SIIVER, et al. (1980). The individualized Educational Plan. Considerations for Preschool Children. Massachusetts. Department of Education.

GINE, C.; ESPUNES, T. y CARASA, P. (1985). Reflexions entorn del concepte de l'excepcionalitat intellectual. En Psicopedagogia de l'excepcionalitat. Barcelona: Fundació Caixa de Pensions.

Ley 13/1982 de 7 de abril de Integración Social de los Minusválidos. BOE de 30 de abril de 1982.

Plan Nacional de Educación Especial. Real Patronato de E.E. Madrid, 1978.

RIBES, E. (1980). Descripción del modelo teórico de campo. Trabajo no publicado.

Ribes, E. (1985). Teoría de la conducta. Un anälisis de campo paramétrico. Méjico: Trillas.

RIBES, E. (1984). Reflexiones sobre el concepto de inteligencia y su desarrollo. En I. Galguera, G. Hinojosa y E. Galindo El retardo en el desamollo. Teoría y práctica. Méjico: Trillas.

Roca, J. (1983). Desenvolupament motor $i$ Psicologia. Barcelona: Generalitat de Catalunya - I.N.E.F.

Ruiz, R. y Gine, C. (1986). Las necesidades Educativas Especiales. Cuademos de Pedagogia, 139, pp. 32-34.

RUIZ, R. (1986). El tractament de les necessitats educatives especials en l'àmbit curricular. En C. Coll Marc curricular per a l'ensenyament obligatori, pp. 83 y ss. Barcelona: Generalitat de Catalunya.

W ARNOCK. H. M. (1978). Report of the Committee of Enquiry into the Education of Handicapped Children and Young People. Londres, Her Majesty's Stationery Office. 